Check for updates

Cite this: RSC Adv., 2018, 8, 18153

Received 12th March 2018

Accepted 4th May 2018

DOI: $10.1039 / \mathrm{c} 8 \mathrm{ra0} 2160 \mathrm{k}$

rsc.li/rsc-advances

\section{An antibacterial collagen membrane crosslinked by the inclusion complex of $\beta$-cyclodextrin dialdehyde and ofloxacin for bacterial keratitis $\dagger$}

\author{
Yawei Chen, ${ }^{\mathrm{ab}}$ Wenjing Song, (D) *ab Xuan Zhao, ${ }^{\text {ab }}$ Qianqian $\mathrm{Han}^{\mathrm{c}}$ and Li Ren (D)*ab
}

Infectious keratitis is one of the leading causes of blindness in the world, especially in developing countries. Corneal transplant surgery is a feasible treatment for bacterial keratitis when drug therapy cannot be effective. However, the amount of corneal donors is far from requirements of clinical treatment and thus, the prognosis of bacterial keratitis is not satisfactory. In this study, we developed a novel antibacterial corneal repair material ( $\beta$-CD-DA/OFLX-Col) for bacterial keratitis. The inclusion complex of $\beta$-cyclodextrin dialdehyde ( $\beta$-CD-DA) with ofloxacin formed by host-guest interaction was used as both a drug vector and a crosslinker for further reaction with the amino groups on lysine of collagen chains. Physical properties, cytocompatibility, and antibacterial property of $\beta$-CD-DA/OFLX-Col film were characterized. The results indicated that the film was mainly transparent and possessed superior mechanical properties. Moreover, human corneal epithelial cells could adhere to the film and proliferate normally, indicating that the $\beta$-CD-DA/OFLX-Col film was non-cytotoxic and had good biocompatibility. Most importantly, the $\beta-C D-D A / O F L X-C o l$ film exhibited prominent antibacterial effects against $E$. coli and $S$. aureus in vitro, which could minimize the risks of infection. The prepared $\beta$-CD-DA/OFLX-Col film could greatly increase bioavailability of drugs and reduce toxic side effects, thus displaying great potential in bacterial keratitis treatment.

\section{Introduction}

Corneal blindness is one of the major causes of visual deficiency after cataract, glaucoma, and age-related macular degeneration. ${ }^{1}$ Keratoplasty is considered to be an effective method to cure corneal blindness. More recently, keratoprosthesis has been developed for more routine cases of corneal blindness where a diseased cornea is replaced with an artificial cornea. However, implantation of an artificial cornea carries a high risk of infection. At present, the common treatment is the frequent use of high-concentration antibiotic eye drops. However, bioavailability of topical antibiotics is poor, especially in the presence of intact corneal epithelium..$^{2-4}$ Once drug treatment is invalid and the infection develops rapidly, it may lead to corneal ulcer perforation and then, therapeutic corneal transplantation will be imperative. Therefore, it is necessary to develop a novel

\footnotetext{
${ }^{a}$ School of Materials Science and Engineering, South China University of Technology, Guangzhou 510641, China.E-mail:phsongwj@scut.edu.cn; psliren@scut.edu.cn

${ }^{b}$ National Engineering Research Center for Tissue Restoration and Reconstruction, Guangzhou 510006, China

'Department of Biomaterials, National Institutes for Food and Drug Control, Beijing 102629, China
}

$\dagger$ Electronic supplementary information (ESI) available. See DOI: $10.1039 / \mathrm{c} 8 \mathrm{ra02160k}$ antibacterial corneal repair material to replace the traditional artificial cornea.

Mehta et al. ${ }^{5}$ developed an anti-infective collagen-based artificial corneal scaffold. Subsequently, it was demonstrated that the addition of vancomycin in a collagen-based artificial corneal scaffold did not significantly alter biomechanical and optical properties of the implant. Moreover, the efficacy of antibiotic-loaded collagen hydrogel in preventing implantable device-associated $S$. aureus infections both in vitro and in vivo was also appreciable. Ren et al. ${ }^{6}$ developed a novel tobramycincontaining antibacterial collagen-based corneal repair material by chemical grafting. The tobramycin-containing collagen film possessed suitable physicochemical properties and showed an excellent antibacterial effect and biocompatibility in vitro and in vivo. However, there are still some problems that need to be solved for collagen-based materials such as poor mechanical performance, ${ }^{7}$ which may lead to an inability to maintain a stable release of the drug. Hence, developing an antibacterial corneal repair material with controllable drug release and excellent mechanical properties is very urgent.

We employed a new biocompatible crosslinking agent $\beta$ cyclodextrin dialdehyde ( $\beta$-CD-DA $)^{8}$ to enhance the mechanical strength of the collagen-based corneal repair material. Moreover, the internal cavity of $\beta$-CD-DA is relatively hydrophobic and can encapsulate a variety of compounds, especially medicines, based on host-guest interactions. ${ }^{9-11}$ Most antibiotic 
drugs used after keratoplasty are hydrophobic and likely to be delivered. Ofloxacin (OFLX) is a highly potent and broadspectrum antibacterial agent used against common Grampositive and Gram-negative pathogens to treat corneal diseases; it is hydrophobic and has poor bioavailability for cornea tissue, and it can be encapsulated by $\beta$-CD-DA.

In this study, we used $\beta$-CD-DA for encapsulating OFLX in the relatively hydrophobic internal cavity of $\beta$-CD-DA through host-guest interactions. The inclusion complex of $\beta$-CD-DA and OFLX ( $\beta$-CD-DA/OFLX) was used to crosslink with collagen, and an ofloxacin-containing antibacterial collagen film ( $\beta$-CD-DA/ OFLX-Col) was prepared for corneal repair. When the antibacterial collagen film was applied onto the surface of the cornea, the encapsulated OFLX could escape from the collagen film through frequent physical shears caused by blinking, thus providing sustained drug release. The physicochemical properties, drug release profile, antibacterial effect, and biocompatibility of the $\beta$-CD-DA/OFLX-Col film were evaluated in vitro.

\section{Materials and methods}

\subsection{Materials}

$\beta$-Cyclodextrin was supplied by Shanghai Bio Science and Technology Co., Ltd., China. Ofloxacin was purchased from Shanghai Macklin Biochemical Co., Ltd, China. 2-Iodoxybenzoic acid (IBX) was provided by Shanghai Aladdin BioChem Technology Co., Ltd., China. Type I collagen (HM Biotech Ltd., Guangzhou, China) was extracted from bovine tendon. Phosphate-buffered saline (PBS) was prepared from Gibco (Thermo Fisher Scientific, USA). Collagenase I from Clostridium histolyticum was supplied by Guangzhou Qiyun Biotechnology Co., Ltd, China. All cell-culture-related reagents were purchased from Sigma Chemical (St. Louis, MO, USA). Deionized water was obtained from a water purification system (Millipore S.A.S., France). Strains of Staphylococcus aureus (S. aureus, strain ATCC 29213) and Escherichia coli (E. coli, strain ATCC 15224) were provided by VWR International, LLC (Radnor, PA, USA).

\subsection{Preparation of inclusion complex}

2.2.1. Preparation of $\boldsymbol{\beta}$-CD-DA. $\beta$-CD-DA was synthesized according to a procedure reported in the literature. ${ }^{\mathbf{8 , 1 2 , 1 3}}$ Briefly, $5.675 \mathrm{~g}$ $\beta$-CD and $3.36 \mathrm{~g}$ IBX were dissolved in $100 \mathrm{~mL}$ DMSO, and the solution was magnetically stirred for $48 \mathrm{~h}$ at $30{ }^{\circ} \mathrm{C}$. Then, the reaction mixture was poured into $900 \mathrm{~mL}$ acetone, stirred for $1 \mathrm{~h}$, and then allowed to stand overnight. After centrifugation, the precipitate was dried under vacuum at $30{ }^{\circ} \mathrm{C}$; next, it was dissolved in water by stirring, and the precipitated impurity was removed by filtration. Finally, lyophilization of the filtrate afforded the desired product (a yield of 93.8\%). ${ }^{1} \mathrm{H}$ NMR $\left(400 \mathrm{MHz}, \mathrm{DMSO}-\mathrm{d}_{6}\right): \delta(\mathrm{ppm})=2.55(\mathrm{DMSO}) ; 3.36\left(\mathrm{H}_{2} \mathrm{O}\right) ; 3.56$ $\left(\mathrm{m}, 14 \mathrm{H}, \mathrm{C}_{2}-\mathrm{H}, \mathrm{C}_{4}-\mathrm{H}\right) ; 3.64\left(\mathrm{~m}, 24 \mathrm{H}, \mathrm{C}_{3}-\mathrm{H}, \mathrm{C}_{5}-\mathrm{H}, \mathrm{C}_{6}-\mathrm{H}\right) ; 4.48(\mathrm{t}$, $\left.5 \mathrm{H}, \mathrm{C}_{6}-\mathrm{OH}\right) ; 4.83\left(\mathrm{~s}, 5 \mathrm{H}, \mathrm{C}_{1}-\mathrm{H}\right) ; 4.93\left(\mathrm{~s}, 2 \mathrm{H}, \mathrm{C}_{1}-\mathrm{H}\right) ; 5.68(\mathrm{~s}, 14 \mathrm{H}$, $\left.\mathrm{C}_{2}-\mathrm{OH}, \mathrm{C}_{3}-\mathrm{OH}\right) ; 9.52(\mathrm{~s}, 1 \mathrm{H}, \mathrm{CHO}) ; 9.70(\mathrm{~s}, 1 \mathrm{H}, \mathrm{CHO})$.

2.2.2. Preparation of inclusion complex of $\beta$-CD-DA/OFLX. The complexes of OFLX with $\beta$-CD-DA were prepared in a mass

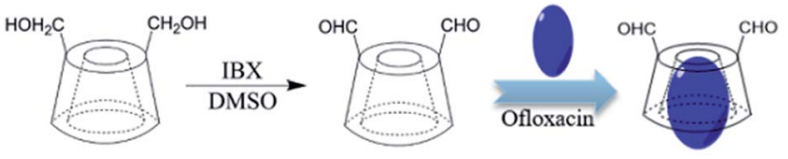

Scheme 1 The synthesis of $\beta$-CD-DA and inclusion complex.

ratio of $1: 10$ by different methods such as coprecipitation, lyophilization, and kneading. For ease of discussion, the optimized samples were prepared by lyophilization. First, $600 \mathrm{mg} \beta$ CD-DA was dissolved in $5.0 \mathrm{~mL}$ deionized water at $50{ }^{\circ} \mathrm{C}$ to obtain a saturated aqueous solution. Then, $60 \mathrm{mg}$ OFLX was suspended in $0.2 \mathrm{~mL}$ ethanol, and this solution was added to the $\beta$-CD-DA solution. The above-mentioned mixed solution was continuously stirred for $1 \mathrm{~h}$ at $200 \mathrm{rpm}$ at $50{ }^{\circ} \mathrm{C}$. The reaction mixture was kept in a refrigerator for $4 \mathrm{~h}$. During this time, a yellow precipitate was formed at the bottom of the bottle. After the mixed solution was lyophilized, the product was dissolved in acetic acid, filtered, and dried in an oven at $50{ }^{\circ} \mathrm{C}$ for $12 \mathrm{~h}$. A yellow powder was eventually obtained. Scheme 1 displays the synthesis of $\beta$-CD-DA and inclusion complex.

\subsection{Characterization of the $\beta$-CD-DA/OFLX inclusion complex}

2.3.1. Fourier transform infrared (FTIR) spectroscopy. The infrared structures of pure $\beta$-CD, $\beta$-CD-DA, OFLX, physical mixture, and inclusion complex were analyzed using Fourier transform infrared spectroscopy (VERTEX 70, Bruker, Germany). Before acquiring an FTIR spectrum of a sample, a background spectrum was collected. All the spectra were obtained from 4000 to $400 \mathrm{~cm}^{-1}$.

2.3.2. Titration of $\boldsymbol{\beta}$-CD-DA. Titration was employed to examine the number of aldehyde groups in each $\beta$-CD-DA molecule. ${ }^{12,14}$ The synthesized $\beta$-CD-DA $(0.05 \mathrm{~g})$ and $30 \mathrm{~mL}$ of $0.01 \mathrm{~mol} \mathrm{~L}^{-1} \mathrm{NaOH}$ solution were added to a conical flask for $4 \mathrm{~min}$ at $70{ }^{\circ} \mathrm{C}$ followed by cooling in ice water. Then, $20 \mathrm{~mL}$ of $0.01 \mathrm{~mol} \mathrm{~L}^{-1} \mathrm{H}_{2} \mathrm{SO}_{4}$ and phenolphthalein indicator were added to the above-mentioned solution, and $0.01 \mathrm{~mol} \mathrm{~L}^{-1} \mathrm{NaOH}$ solution was added until the solution turned red.

2.3.3. Powder X-ray diffraction pattern (PXRD). X-Ray diffractograms of OFLX, $\beta$-CD-DA, physical mixture, and inclusion complex after lyophilization were obtained using an X-ray diffractometer (Empyrean, PANalytical, Netherlands), and the samples were investigated in the $2 \theta$ range of $5-50^{\circ}$.

2.3.4. 2D ${ }^{1}$ H-NMR measurements. Two-dimensional rotating-frame Overhauser effect spectroscopy (ROESY) experiments were performed using a BRUKER-NMR $400 \mathrm{MHz}$ instrument operating at 298 K (AVANCE111 400, Bruker, Switzerland), and the standard Bruker program was used with $\mathrm{D}_{2} \mathrm{O}$ as a solvent. The ROESY spectrum for the $\beta$-CD-DA/OFLX mixture was recorded under the spin-lock condition by acquisition of 1024 (F2) × 64 (F1) complex data points. The ROESY data were acquired in a phase-sensitive mode with a mixing time of $0.2 \mathrm{~s}$ and a relaxation delay of $2 \mathrm{~s}$. 

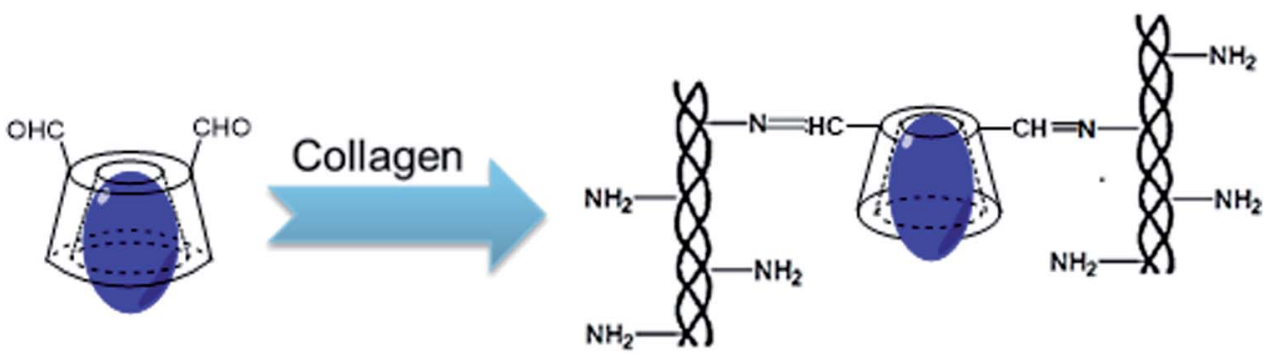

Scheme 2 The chemical reaction mechanism between collagen and $\beta-C D-D A / O F L X$.

2.3.5. Inclusion rate of $\boldsymbol{\beta}$-CD-DA/OFLX. The inclusion rate is an important index of the inclusion efficiency, and the higher the inclusion rate, the better the inclusion effect. The loading mass of OFLX was determined by a UV-vis spectrophotometer. The UV spectra of the inclusion complex, pure OFLX, and $\beta$-CD-DA in deionized water $\left(50 \mu \mathrm{g} \mathrm{mL} \mathrm{m}^{-1}\right)$ were obtained by a NanoDrop-2000 UV-vis spectrophotometer (Unico, Shanghai, China). The scans were registered from 200 to $400 \mathrm{~nm}$. The detection wavelength of OFLX in the clathrate was determined after determining the characteristic absorption peak of OFLX. Then, the right amount of OFLX was diluted with deionized water to obtain the final solution concentrations of $5,10,15,20,25,30,35$, and $40 \mu \mathrm{g} \mathrm{mL}{ }^{-1}$. The absorbance was measured at the detection wavelength, and the mass concentration was used to draw a standard curve of the absorbance. Then, the quantity of OFLX in the clathrate was calculated by the linear equation, and the inclusion rate (\%) was calculated by using the following equations:

$$
\begin{gathered}
R(\%)=(C \times 50 \times 10) /(m \times X) \times 100 \% \\
X(\%)=\left(n_{1} \times 361\right) /\left(n_{1} \times 361+n_{2} \times 1131\right) \times 100 \%
\end{gathered}
$$

Here, $R$ is the inclusion rate (\%), $C$ is the concentration of OFLX $\left(\mu \mathrm{g} \mathrm{mL}{ }^{-1}\right), m$ is mass of the inclusion complex $(\mu \mathrm{g}), X$ is the quantity percentage of OFLX (\%), $n_{1}$ is amount of OFLX before inclusion, $n_{2}$ is amount of $\beta$-CD-DA before inclusion, and 1131 and 361 indicate molecular weights of $\beta$-CD-DA and OFLX, respectively.

\subsection{Preparation of collagen films}

Type I collagen solution $\left(100 \mathrm{~mL}, 6.5 \mathrm{mg} \mathrm{mL}^{-1}\right)$ was prepared by dissolving type I collagen in $0.01 \mathrm{~mol} \mathrm{~L}^{-1} \mathrm{HCl}$ solution at $4{ }^{\circ} \mathrm{C}$. The inclusion complex was dissolved in deionized water to achieve the concentration of $26 \mathrm{mg} \mathrm{mL}^{-1}$ and then, it was added to the collagen solution. The mixed solution ( $\beta$-CD-DA/ OFLX : collagen $=1: 5, \mathrm{w} / \mathrm{w}$ ) was thoroughly stirred at $4{ }^{\circ} \mathrm{C}$ for $24 \mathrm{~h}$ to obtain homogeneous blending and crosslinking. Then, the cross-linked collagen solution was dispensed into a specific mold and dried to form a cornea-shaped film. After that, the $\beta$ CD-DA/OFLX-Col film was rinsed three times with deionized water and dried again. For comparison, the collagen films crosslinked by EDC/NHS, ${ }^{15} \beta$-CD-DA without OFLX, and a $\beta$-CDDA-OFLX physical mixture were also prepared as the control group (Scheme 2).

\subsection{Characterization of physical and chemical properties of collagen films}

2.5.1. Macroscopic and microscopic morphology. After soaking the membrane in PBS for $1 \mathrm{~h}$, the membrane was imaged using a digital camera (Nikon D3100) to investigate its macroscopic morphology. Sectional morphology of the membrane was observed using a scanning electron microscope (SEM, MERLIN, Zeiss) after the dried membrane was brittle fracture in liquid nitrogen.

2.5.2. Water content analysis. Water absorptions of the collagen films crosslinked by EDC/NHS (EDC/NHS-Col), $\beta$-CDDA ( $\beta$-CD-DA-Col), and $\beta$-CD-DA/OFLX ( $\beta$-CD-DA/OFLX-Col) were measured by swelling them in PBS $(\mathrm{pH} 7.4)$ at room temperature. The unabsorbed water on the surface of the films was removed gently, and the wet weight of each sample $\left(M_{t}\right)$ was immediately recorded. Then, the films were vacuum-dried to obtain a constant weight $\left(M_{0}\right)$. The water contents of the films were calculated according to the following equation: $W_{t}=\left(M_{t}-\right.$ $\left.M_{0}\right) / M_{t} \times 100 \%$.

2.5.3. Light transmittance. Before conducting the transparency test, the films were immersed in PBS for more than $2 \mathrm{~h}$ to uptake water until saturation and then, the residual solutions on the surfaces of the EDC/NHS-Col, $\beta$-CD-DA-Col, and $\beta$-CDDA/OFLX-Col films were absorbed by filter papers. After that, the films were fixed into a specimen chamber of a UV3802 ultraviolet-visible spectrophotometer (Shanghai UNICO, China). Light transmittance of the films was quantitatively evaluated by visible-light transmission measurements.

2.5.4. Stability of collagen films. The resistance of the collagen-based films to collagenase was evaluated as described previously. ${ }^{16}$ The samples weighing approximately $50 \mathrm{mg}$ were equilibrated for $1 \mathrm{~h}$ in $5 \mathrm{~mL}$ of $0.1 \mathrm{M}$ Tris-HCl buffer (pH 7.4) containing $5 \mathrm{mM} \mathrm{CaCl}_{2}$ at $37^{\circ} \mathrm{C}$. Subsequently, $1 \mathrm{mg} \mathrm{mL}^{-1}(288$ $\mathrm{U} \mathrm{mL}^{-1}$ ) collagenase solution was added to give a final collagenase concentration of $5 \mathrm{U} \mathrm{mL}^{-1}$. The collagenase solution was replaced every $8 \mathrm{~h}$ to maintain the collagenase activity. At various time intervals, the samples were removed from the solution, gently blotted on filter papers, and weighed. All 
samples were tested in triplicate. The percent residual mass of the sample was calculated according to the equation residual mass $\%=W_{t} / W_{0} \times 100 \%$, where $W_{0}$ represents the initial weight of the films, and $W_{t}$ is the weight of the films at each time point.

2.5.5. Mechanical test. Analyses of the mechanical properties of the samples were conducted using a uniaxial load testing instrument (Model \#5567, Instron Corporation, Issaquah, WA, USA) equipped with a load cell of $10 \mathrm{~N}$ capacity at a crosshead speed of $10 \mathrm{~mm} \mathrm{~min}{ }^{-1}$ and an initial grip separation of $10 \mathrm{~mm}$. Before the test, the films were immersed in PBS for $1 \mathrm{~h}$ and then, they were cut into dumbbell-shaped specimens of identical rectangular gage areas (width, $5 \mathrm{~mm}$; length, 10 $\mathrm{mm}$; and thickness, $0.3 \mathrm{~mm}$ ) with two $8 \mathrm{~mm}$ end tabs.

\subsection{Assessment of drug release in vitro}

OFLX release trials were performed in vitro in a constant temperature shaker (Forma481, Thermo life sciences, USA) at $37^{\circ} \mathrm{C}$. For the trials, $400 \mathrm{mg}$ of $\beta$-CD-DA/OFLX-Col and $\beta$-CD-DAOFLX-Col films was immersed in $3 \mathrm{~mL}$ of PBS ( $\mathrm{pH} 7.4$ ) and then, all of the leached liquor was collected at regular time intervals $(1,2,4,8,12,24,48,72,96,120,144$, and $168 \mathrm{~h})$. The volume of the medium was kept constant by adding the same amount of PBS. The concentrations of OFLX were measured using a quantified method described in Section 2.3.5. The percentage of OFLX release was calculated by dividing the amount of drug in the release medium by total OFLX loading on the films. The experiments of OFLX release were carried out in triplicate.

\subsection{Cytocompatibility in vitro}

2.7.1. Corneal epithelial cell culture. Human corneal epithelial cells (HCECs) were obtained from the State Key Laboratory of Ophthalmology, Zhongshan Ophthalmic Center, Sun Yat-sen University, China. The cells were cultured in high glucose Dulbecco's modified Eagle's medium (DMEM; Gibco by Thermo Fisher Scientific, USA) with 15\% fetal bovine serum (FBS; Gibco BRL), $5 \mu \mathrm{g} \mathrm{mL}^{-1}$ recombinant human insulin (Sigma-Aldrich, USA), $5 \mu \mathrm{g} \mathrm{mL}^{-1}$ human transferrin (SigmaAldrich, USA), $10 \mathrm{ng} \mathrm{mL}^{-1}$ human epidermal growth factor (hEGF; Cell Signaling Technology, USA), 1\% non-essential amino acid (NEAA; Gibco by Life Technologies, USA), and 1\% penicillin/streptomycin (P/S; Gibco by Life Technologies, USA). HCECs were grown in an RS biotech chamber containing 5\% $\mathrm{CO}_{2}$ at $37{ }^{\circ} \mathrm{C}$, and confluent cells were subcultured every 2-3 days by trypsinization with $0.25 \%$ TrypsinEDTA (Gibco by Life Technologies, USA). The cell supernatant was replaced every other day to maintain an adequate supply of cell nutrients.

2.7.2. The response of HCECs to collagen films. Before the cell experiments, the $\beta$-CD-DA-Col and $\beta$-CD-DA/OFLX-Col films were sterilized by ultraviolet radiation for $2 \mathrm{~h}$ and then, they were washed three times with PBS under aseptic conditions. After a series of treatments, the films were transferred to a 48well tissue culture plate (Corning, UK). Then, the cell suspension was inoculated into the set orifice plates with a density of 1 $\times 10^{4}$ cells per $\mathrm{mL}$. The cell-seeded films were then incubated in the RS biotech chamber $\left(5 \% \mathrm{CO}_{2}, 37^{\circ} \mathrm{C}\right)$. The culture medium was replaced every 2 days. The response of HCECs to the films and the morphology of HCECs were examined. Before observation with an Eclipse Ti-U inverted fluorescence microscope (Nikon, Japan), the surfaces of the films were washed with PBS.

2.7.3. The proliferation of HCECs on collagen films. After the samples were transferred to the 48-well tissue culture plates (Corning, UK), HCEC suspensions were seeded onto the $\beta$-CD-DA/OFLX-Col films (experimental group, $n=5$ ) and the $\beta$-CD-DA-Col films (control group, $n=5$ ). The seeded cell density was $1 \times 10^{4}$ cells per mL. After 1,3 , and 5 days, the proliferation activity of HCECs on the films was determined by a CCK-8 assay (Dojindo, Kumamoto, Japan) with a Thermo3001 microplate reader (Thermo, USA) using an optical density (OD) of $450 \mathrm{~nm}$.

\subsection{Antibacterial test}

2.8.1. Bacterial culture. In bacterial keratitis, Grampositive cocci and Gram-negative bacilli are predominant. ${ }^{17}$ In this study, therefore, the antibacterial activities of the films were evaluated by revised filter paper disk diffusion method, and $S$. aureus and $E$. coli were used, which are representatives of Gram-positive bacteria and Gram-negative bacteria, respectively. $S$. aureus and $E$. coli were grown in LB broth $(1 \% \mathrm{w} / \mathrm{v}$ tryptone, $0.5 \% \mathrm{w} / \mathrm{v}$ yeast extract, and $0.5 \% \mathrm{w} / \mathrm{v} \mathrm{NaCl}$ ) at $37{ }^{\circ} \mathrm{C}$ with constant agitation under aerobic conditions before any further experiment.

2.8.2. Zone of inhibition. The $\beta$-CD-DA-Col and $\beta$-CD-DA/ OFLX-Col films were tested on two pathogenic bacteria using a zone of inhibition assay in LB agar. Bacterial number of a $48 \mathrm{~h}$ culture in LB broth was estimated by comparing the absorbance at $600 \mathrm{~nm}$ using a Beckman DU-40 spectrophotometer (Fullerton, CA). The concentration was then adjusted to $1-5 \times 10^{9}$ CFU mL ${ }^{-1}$ using PBS (pH 7.4), and $100 \mu \mathrm{L}$ bacterial suspension was evenly spread on the agar plates using a sterile plastic rod spreader. The agar plates were dried at room temperature for $24 \mathrm{~h}$. Sterilized filter paper disks (Jinton, Tianjin, China, $10 \mathrm{~mm}$ in diameter) were arranged on the plates, and $20 \mu \mathrm{L}$ volume of test solutions (one for OFLX dispersed in deionized water evenly and another for the $\beta$-CD-DA/OFLX inclusion complex dissolved in deionized water) was added to the paper disks (positive control). The $\beta$-CD-DA-Col and $\beta$-CD-DA/OFLX-Col films $(10 \mathrm{~mm}$ in diameter) were placed onto the agar surface (one for negative control and the other for testing). After the plates were incubated at $37{ }^{\circ} \mathrm{C}$ for $24 \mathrm{~h}$, the diameters of the distinctly clear zones were measured using a metric ruler. The zone of inhibition experiment was repeated four times, and the data were statistically analyzed using Duncan's multiple range test.

2.8.3. Quantitative experiment of antibacterial activity. Bacterial number of a 48 h culture in LB broth was estimated by comparing the absorbance at $600 \mathrm{~nm}$ using a Beckman DU-40 spectrophotometer (Fullerton, CA). The concentrations of $S$. aureus and $E$. coli were then adjusted to $5 \times 10^{9}$ and $6 \times 10^{9} \mathrm{CFU}$ $\mathrm{mL}^{-1}$ using PBS ( $\mathrm{pH} 7.4$ ), corresponding to the OD values of 0.320 and 0.404 , respectively. The $\beta$-CD-DA-Col and $\beta$-CD-DA/ OFLX-Col films were placed in the 48 -well plate, covered with $0.5 \mathrm{~mL}$ bacterial suspension, and incubated for $24 \mathrm{~h}$ at $37^{\circ} \mathrm{C}$. Then, the bacterial suspension and the samples were 
A

A

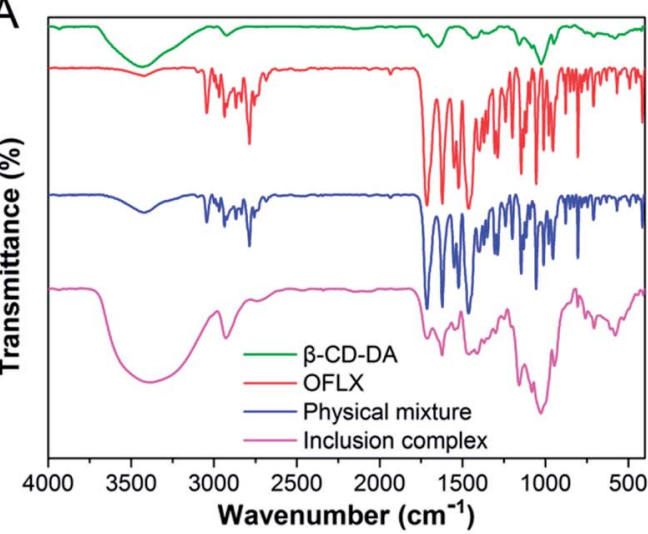

B

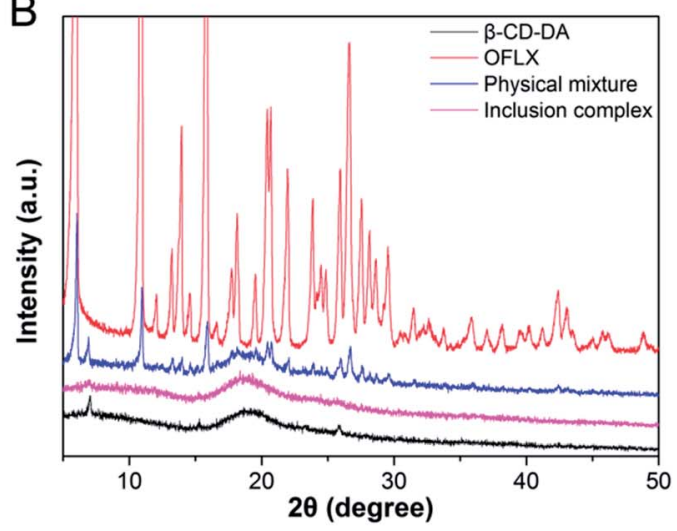

Fig. 1 FTIR (A) and PXRD (B) spectra of $\beta$-CD-DA, OFLX, physical mixture, and inclusion complex.

transferred to centrifuge tubes together, and the tubes were shaken for 10 seconds and then, the OD values were obtained for two transferred pathogenic bacterial suspension in the 96well plate.

\section{Results and discussion}

\subsection{Analysis of $\beta$-CD-DA/OFLX inclusion complex}

3.1.1. FTIR spectra analysis. FTIR characterization was used to identify the formation of the inclusion complexes based on the change in peak shape, peak position, and peak intensity in the front and rear of the guest clathrated by the host. FTIR spectra of $\beta$-CD-DA, OFLX, physical mixture ( $1: 1)$, and inclusion complexes are shown in Fig. 1A. The spectra of $\beta$-CD-DA and inclusion complex were similar, indicating that the skeletal structure of $\beta$-CD-DA had not changed. However, the peak of hydroxyl groups shifted from $3435 \mathrm{~cm}^{-1}$ to $3381 \mathrm{~cm}^{-1}$, indicating that the environment of $\beta$-CD-DA had changed, which caused the displacement of the hydroxyl telescopic peak. In the IR spectrum of OFLX, the peak at $3422 \mathrm{~cm}^{-1}$ was assigned to the hydroxyl groups, and the interactions of intermolecular hydrogen bonds formed a wide band. The peak at $1714 \mathrm{~cm}^{-1}$ was assigned to the vibration of the carboxyl group of OFLX, and this characteristic peak was also observed for the physical mixture and inclusion complexes. Furthermore, the peak at $1056 \mathrm{~cm}^{-1}$ was assigned to the telescopic vibration of C-F of OFLX, but this peak was not present for the inclusion complexes, thus showing that there were interactions between $\beta$-CD-DA and OFLX.

3.1.2. XRD analysis. Lack of crystallinity was another evidence for the formation of the inclusion complex. ${ }^{18}$ Fig. $1 \mathrm{~B}$ shows the XRD patterns of the guest (OFLX), host ( $\beta$-CD-DA), and their complex systems. $\beta$-CD-DA had no obvious crystal peaks, which indicated that it was an amorphous powder. The powder X-ray diffraction pattern of OFLX revealed several sharp and high-intensity peaks at different diffraction angles $(2 \theta)$ of $6.02,10.92,13.95,15.94,20.42,21.96$, and $26.60^{\circ}$, suggesting that OFLX had crystalline nature. Furthermore, the OFLX-specific peaks remained apparent for the $\beta$-CD-DA and OFLX physical mixture. However, the $\beta$-CD-DA/OFLX inclusion complex showed a similar crystalline diffractogram with that of $\beta$-CD-DA. Moreover, the partial diffraction peaks of OFLX still existed, indicating that the inclusion complex was not a simple mechanical mixture but rather a new substance. The drug did not enter the cavity completely.

3.1.3. 2D ${ }^{1} \mathbf{H}$ NMR (ROESY) studies. The highfield chemical shift changes in $\beta$-CD-DA cavity proton signals, namely $\mathrm{H}-3^{\prime}$ and $\mathrm{H}-5^{\prime}$ (Fig. 2A), in the presence of the guest confirmed the formation of the inclusion complex, ${ }^{19}$ and the part of the guest molecule encapsulated by $\beta$-CD-DA was identified by ROESY spectroscopy. ${ }^{20}$ To study the geometry of the inclusion complex of OFLX with $\beta$-CD-DA, 2D ROESY NMR experiment was used to examine the configuration of OFLX in the $\beta$-CD-DA cavity. Fig. $2 \mathrm{C}$ shows a partial contour plot of the ROESY spectra of the inclusion complex; there were several intermolecular cross-peaks between $\mathrm{H}-12$ and $\mathrm{H}-27$ of OFLX and $\mathrm{H}-3^{\prime}$ and $\mathrm{H}-5^{\prime}$ of $\beta-\mathrm{CD}-\mathrm{DA}$, which might indicate that the matrix of the guest molecules entered the cavity of $\beta$-CD-DA from big ring-edge side of $\beta$-CD-DA, leaving piperazine ring out of $\beta$-CD-DA cavity. ${ }^{21}$ Combining the
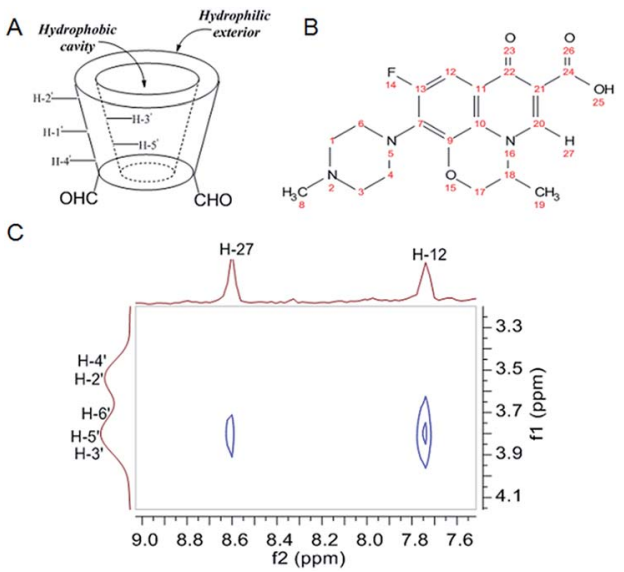

Fig. 2 (A) Truncated cone shape of $\beta-C D-D A$ and position of its different protons. The hydrophobic cavity of $\beta-C D-D A$ is lined with $\mathrm{H}$ $3^{\prime}$ and $\mathrm{H}-5^{\prime}$ protons and their NMR signals are more influenced than those of the other $\beta-C D-D A$ protons $\left(H-1^{\prime}, 2^{\prime}, 4^{\prime}, 6^{\prime}\right)$; (B) chemical structure of OFLX; (C) the 2D ${ }^{1} \mathrm{H}$ NMR ROESY partial counter plot spectra of $\beta$-CD-DA/OFLX inclusion complex in $\mathrm{D}_{2} \mathrm{O}$ at $298 \mathrm{~K}$. 


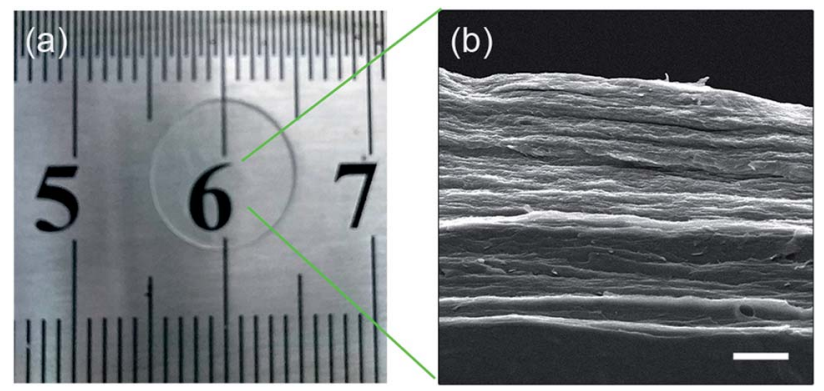

Fig. 3 The image of $\beta$-CD-DA/OFLX film after absorbing water (a) and SEM photo of the section (b); scale bar: $10 \mu \mathrm{m}$.

FTIR and XRD spectra, it could be concluded that OFLX was closely included into the inner cavity of $\beta$-CD-DA to form a supramolecular system.

\subsection{Analysis of physical and chemical properties of collagen} films

3.2.1. Macroscopic and microscopic morphology. The digital image of the membrane after immersing in PBS for $1 \mathrm{~h}$ is shown in Fig. 3a. After storing the sample in PBS for $1 \mathrm{~h}$, it was found that the water absorption of the film tended to be constant. The membrane had a smooth and transparent appearance. Fig. $3 \mathrm{~b}$ shows the sectional morphology of the membrane. The $\beta$-CD-DA/OFLX film showed a layered laminar structure similar to the structure of natural cornea.

3.2.2. Water absorption. The water content data are shown in Fig. 4A. The water absorption of the $\beta$-CD-DA/OFLX-Col film $(78.0 \pm 1.1 \%)$ was close to that of the $\beta$-CD-DA-Col film $(74.4 \pm$ $0.7 \%$ ), indicating that the addition of OFLX did not have a significant impact on the effect of crosslinker. Moreover, the water content of the $\beta$-CD-DA/OFLX-Col film was quite similar to that of human cornea $(78.0 \pm 3.0 \%),{ }^{22}$ and it was significantly lower than that of the EDC/NHS-Col film $(86.0 \pm 1.2 \%)(n=10$, $p<0.05)$. The reduction of water content was probably due to the improvement in crosslinking degree, and crosslinking density led to more rigid network structures. Therefore, both relaxation of polymer chains and water uptake ability of the films were restricted. ${ }^{23}$

3.2.3. Light transmittance. Fig. $4 \mathrm{~B}$ shows the light transmittance curves of the EDC/NHS-Col, $\beta$-CD-DA-Col, and $\beta$-CDDA/OFLX-Col films. The thickness of the wet samples was about $500 \pm 20 \mu \mathrm{m}$. The transparency of $\beta$-CD-DA/OFLX-Col film was slightly different from EDC/NHS-Col film; also, the transparency of $\beta$-CD-DA/OFLX-Col film was slightly higher than EDC/NHS-Col film. With an increase in wavelength, the light transmittance of the films increased to their maximum (close to $85 \%)$. In addition, the transparencies of the $\beta$-CD-DA/OFLX-Col and $\beta$-CD-DA-Col films were basically the same, and they were within the 600-800 $\mathrm{nm}$ range, which indicated that the addition of drugs did not affect the transmittance of the membrane.

3.2.4. Mechanical property. The corneal repair material should have a proper mechanical strength so that it can withstand the stitching of the surgical suture to keep the material intact. A comparison between the films showed that their mechanical properties were quite different (Fig. $5, n=5$ ). The thickness of the wet films was about $500 \pm 20 \mu \mathrm{m}$. The ultimate tensile stresses of EDC/NHS-Col, $\beta$-CD-DA-Col, and $\beta$-CD-DA/ OFLX-Col were $1.167 \pm 0.035,2.018 \pm 0.030$, and $1.988 \pm$ $0.034 \mathrm{MPa}$, respectively (Fig. 5A). The films crosslinked by $\beta$-CDDA had a higher tensile strength, and they demonstrated a better cross-linking effect than EDC/NHS. The elongation at break values of $\beta$-CD-DA/OFLX-Col and $\beta$-CD-DA-Col were 17.04 $\pm 1.56 \%$ and $16.89 \pm 1.58 \%$, respectively, and the values were significantly lower than that of EDC/NHS-Col $(48.62 \pm 3.69 \% ; p$ $<0.01$ ) (Fig. 5B). Because of the high tensile strength and low elongation at break, $\beta$-CD-DA/OFLX-Col (11.67 $\pm 0.159 \mathrm{MPa})$ and $\beta$-CD-DA-Col $(11.95 \pm 0.162 \mathrm{MPa})$ had a very high elastic moduli, which were much higher than that of EDC/NHS-Col $(2.38 \pm 0.150 \mathrm{MPa} ; p<0.01)$ (Fig. 5C). The $\beta$-CD-DA/OFLX-Col film exhibited suitable mechanical properties compared with the EDC/NHS-Col film, and the addition of drugs had a negligible impact on the mechanical properties of the membrane.

\subsection{Collagenase degradation in vitro}

As shown in Fig. 6A, in general, an increase in crosslinking degree improves collagenase resistance in vitro. The
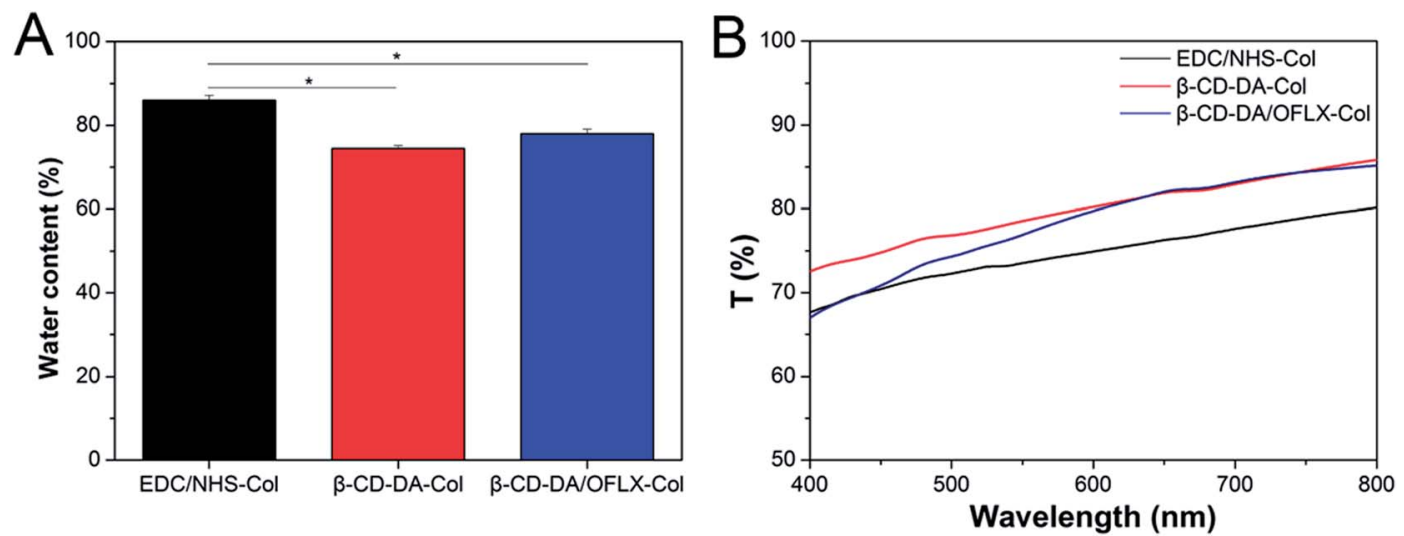

Fig. 4 Water absorption percentages (A) and optical properties (B) of EDC/NHS-Col, $\beta$-CD-DA-Col and $\beta$-CD-DA/OFLX-Col films. 

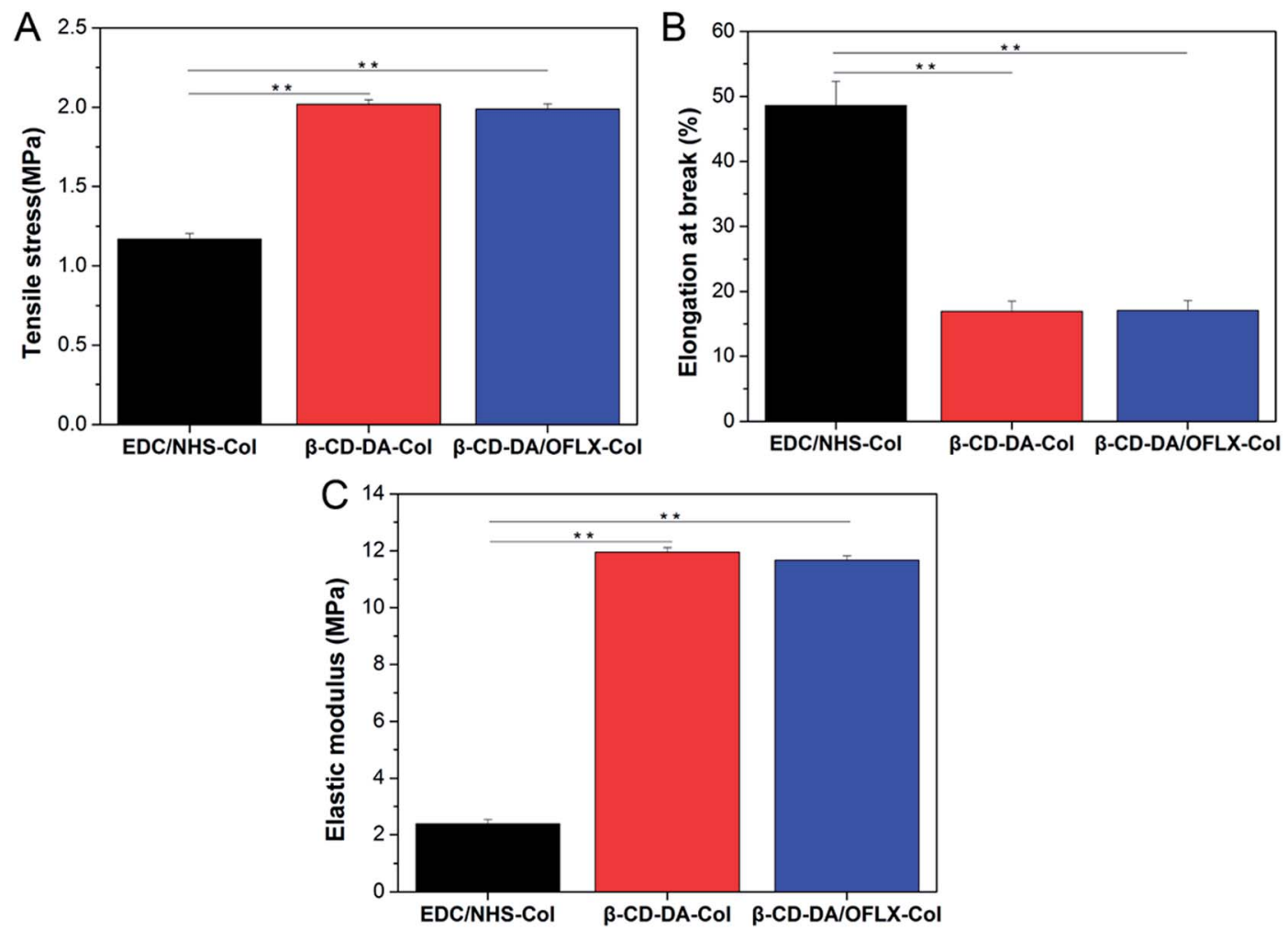

Fig. 5 Mechanical properties of EDC/NHS-Col, $\beta$-CD-DA-Col and $\beta$-CD-DA/OFLX-Col films. (A) Tensile stress; (B) elongation at break; (C) elastic modulus.

degradation of the collagen films crosslinked with EDC/NHS was completed by about $32-36 \mathrm{~h}$. The degradation of the collagen films crosslinked with $\beta$-CD-DA and $\beta$-CD-DA/OFLX required more than $48 \mathrm{~h}$ to complete. Our present results showed that the $\beta$-CD-DA crosslinked films had better antienzymatic stability than the EDC/NHS-crosslinked ones. The high crosslinking efficiency between $\beta$-CD-DA and collagen was due to strong interactions, which was the form of covalent bond between aldehyde group in $\beta$-CD-DA and amino group of collagen, and strong hydrogen bonds could be formed between hydroxyl and carboxyl groups, which significantly improved the resistance of the collagen-based films to collagenase.

\subsection{OFLX release in vitro}

The results of the investigation of OFLX release (the cumulative release in vitro) from the collagen films crosslinked by $\beta$-CD-DA in the form of the physical mixture and inclusion complex are shown in Fig. 6B. The release profiles of both conditions exhibited an exponential tendency. The burst release of OFLX from the $\beta$-CD-DA/OFLX-Col inclusion complex in the first $16 \mathrm{~h}$ was about $30 \%$ of the total OFLX loading amount, and this was
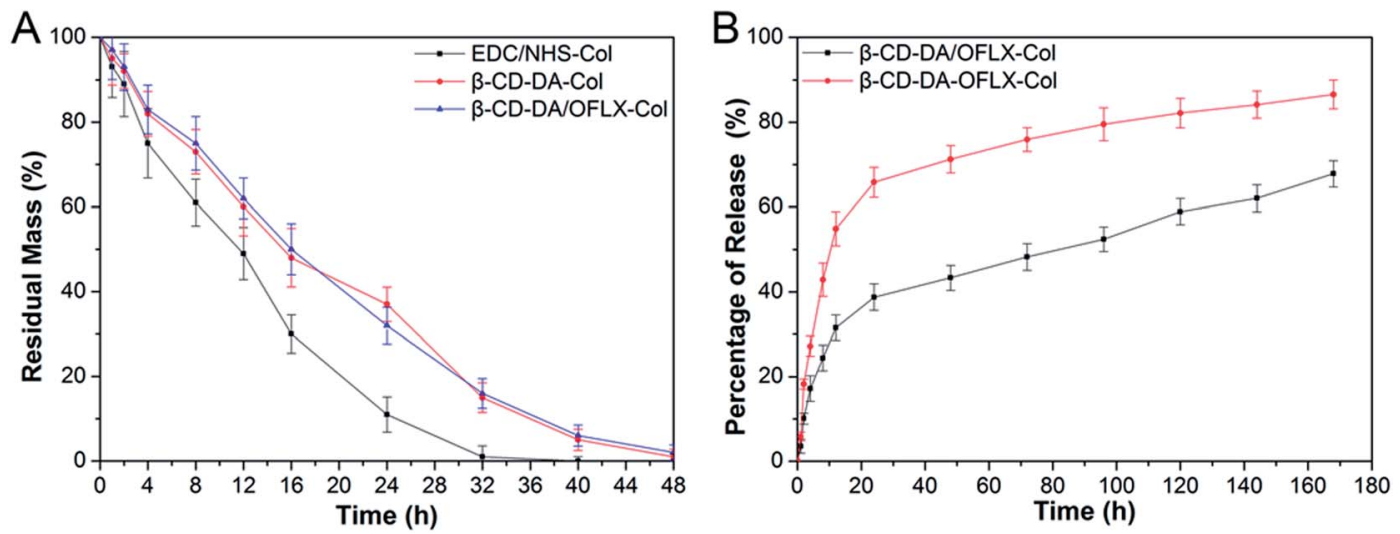

Fig. 6 (A) In vitro collagenase biodegradation of EDC/NHS-Col, $\beta$-CD-DA-Col, and $\beta$-CD-DA/OFLX-Col films. (B) Cumulative release of OFLX from $\beta$-CD-DA/OFLX-Col inclusion complex films and $\beta$-CD-DA-OFLX-Col physical mixture films. 

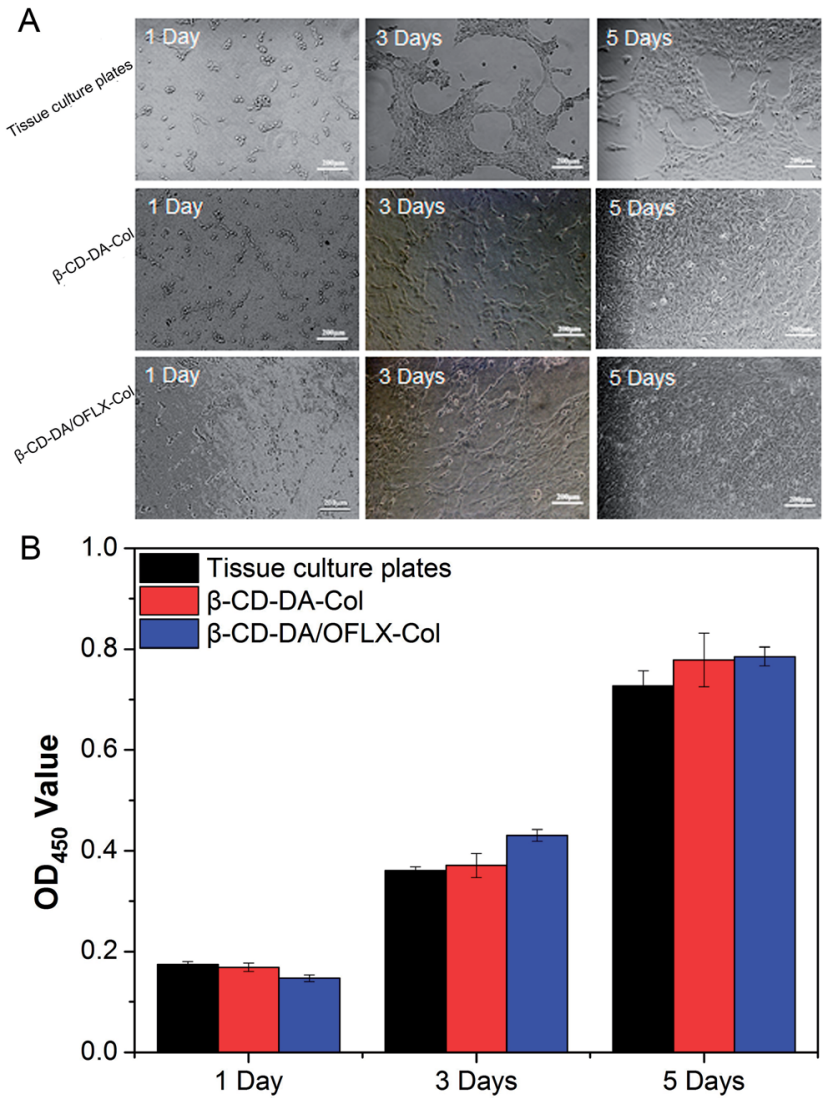

Fig. 7 Morphology of HCECs seeded (A) and OD values (B) for tissue culture plates, $\beta$-CD-DA-Col film and $\beta$-CD-DA/OFLX-Col film for 1 day, 3 days and 5 days.

followed by a slower release of the drug. By contrast, the release profiles of collagen films crosslinked by $\beta$-CD-DA in the form of physical mixture of drugs were even more pronounced, and nearly $60 \%$ of OFLX was released in the first $16 \mathrm{~h}$. On day 7 , the total OFLX released from the physical mixture film was $90 \%$, whereas that released from the inclusion complex film was less than $70 \%$. Thus, the inclusion complex film could be used for
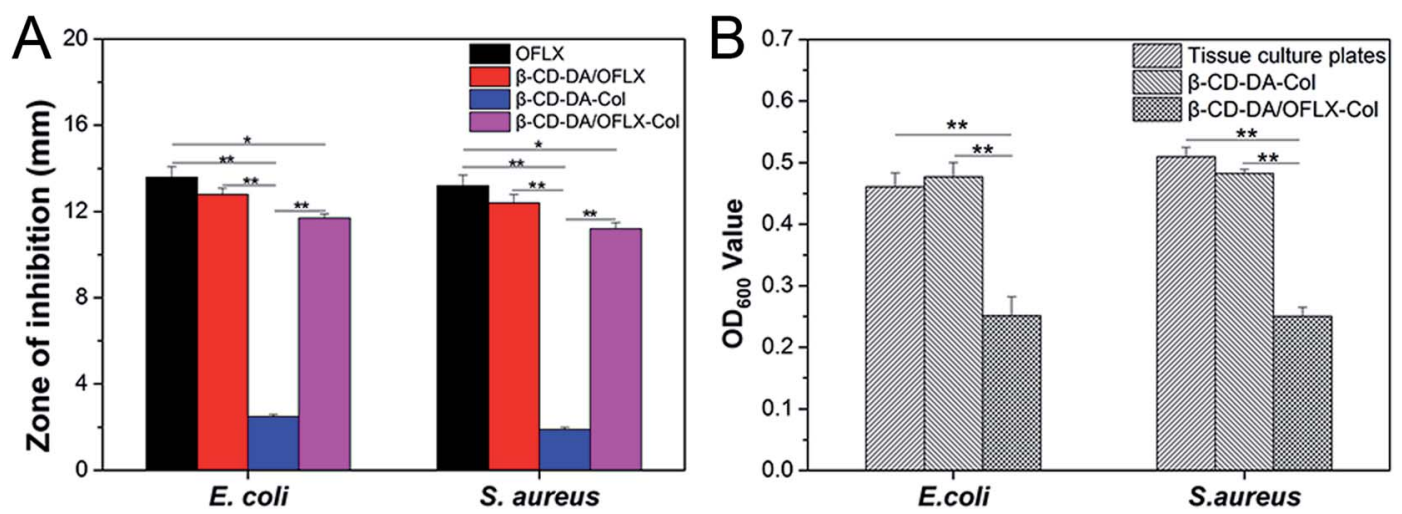

Fig. 8 (A) Zone of inhibition of OFLX, $\beta$-CD-DA/OFLX, $\beta$-CD-DA-Col film, and $\beta$-CD-DA/OFLX-Col film against E. coli and S. aureus. (B) Bacteriostasis results of tissue culture plates, $\beta-C D-D A-C o l$ film, and $\beta-C D-D A / O F L X-C o l$ film against $E$. coli and $S$. aureus. a long-term drug release, which can ensure a certain antimicrobial activity.

\subsection{The morphology of HCECs}

Fig. 7A shows the morphologies of HCECs on tissue culture plates and $\beta$-CD-DA-Col and $\beta$-CD-DA/OFLX-Col films at different time points. At day 1, the seeded cells had adhered to the surface of the film quite well, and there was no significant difference in the number of cells among them. At day 3, the morphology of HCECs gradually changed from a round shape to a spindle shape on two films, which was quite similar to that of HCECs growing on normal corneal tissues. ${ }^{24}$ Also, more roundshaped cells were observed on the tissue culture plates in comparison with those observed on the films, indicating that the seeded HCECs adhered better to the collagen films than tissue culture plates. After HCECs were seeded on the films, the cells proliferated rapidly. After 5 days, it was found that the films were almost completely covered with HCECs.

In the field of tissue engineering, one of the important steps in the development of a novel scaffold is the evaluation of cytotoxicity. The CCK-8 test (Fig. 7B) showed the proliferation of HCECs on tissue culture plates and $\beta$-CD-DA-Col and $\beta$-CD-DA/ OFLX-Col films. There was no significant difference in the toxicities of the three materials, indicating that the collagen membrane had excellent biocompatibility, and the addition of drugs did not increase the toxicity of the films.

\subsection{Anti-bacterial effect}

The antibacterial activities of the $\beta$-CD-DA/OFLX-Col films were analyzed against both Gram-positive and Gram-negative bacteria, and the results are presented in Fig. 8A and B. It can be seen from Fig. 8A that the $\beta$-CD-DA-Col film without OFLX failed to show any activity against $E$. coli $(2.5 \pm 0.1 \mathrm{~mm})$ and $S$. aureus $(1.9 \pm 0.1 \mathrm{~mm})$. The highest antimicrobial activity, which was obtained with the samples of pure OFLX, was due to their direct contact with the tested bacteria, and the inhibition zone diameters were $13.6 \pm 0.5$ and $13.2 \pm 0.5 \mathrm{~mm}$. For the $\beta$-CD-DA/ OFLX-Col film, the maximum zone of inhibition of $11.7 \pm$ $0.2 \mathrm{~mm}$ was found against $E$. coli, and the $\beta$-CD-DA/OFLX 
inclusion complex showed the maximum $12.8 \pm 0.3 \mathrm{~mm}$ zone of inhibition against $E$. coli. Also, the maximum zone of inhibition of $11.2 \pm 0.3 \mathrm{~mm}$ was found for the $\beta$-CD-DA/OFLX-Col film, and the maximum zone of inhibition of $12.4 \pm 0.4 \mathrm{~mm}$ was observed for the $\beta$-CD-DA/OFLX inclusion complex against $S$. aureus. The difference in the size of zones among the $\beta$-CD-DA-Col film, pure OFLX, $\beta$-CD-DA/OFLX inclusion complex, and $\beta$-CD-DA/ OFLX-Col film was significant $(p<0.01)$. Additionally, there were significant differences in the inhibition zones of pure OFLX and the $\beta$-CD-DA/OFLX-Col film $(p<0.05)$. OFLX is one of the newer quinolone antibiotics with a broad spectrum of antibacterial activity. ${ }^{25}$ In the results of the inhibition zone, the bacteriostasis effect on $E$. coli and $S$. aureus was basically consistent with the quantitative OFLX.

Fig. $8 \mathrm{~B}$ shows the bacteriostasis effects of tissue culture plates, $\beta$-CD-DA-Col film and $\beta$-CD-DA/OFLX-Col film in $24 \mathrm{~h}$. Compared to the tissue culture plates and $\beta$-CD-DA-Col film, the $\beta$-CD-DA/OFLX-Col film exhibited significant difference in antimicrobial activity $(p<0.01)$. The results indicated that the $\beta$-CD-DA/OFLX-Col film had a remarkable antibacterial effect, which was enough to inhibit most Gram-positive and negative bacteria, even though the amount of drug release was less than $40 \%$ in $24 \mathrm{~h}$. Therefore, this film not only helps avoid the trouble of frequent drug use, but also reduces the chance of drug resistance, and it can be effective for a long period of time.

\section{Conclusions}

In summary, we have successfully prepared $\beta$-CD-DA biocrosslinker and $\beta$-CD-DA/OFLX inclusion complex by a convenient and efficient method. Then, $\beta$-CD-DA/OFLX was used to crosslink with collagen, and the properties of cross-linked collagen improved significantly. The test results suggested that the introduction of $\beta$-CD-DA could promote light transmittance and mechanical properties of the collagen-based corneal repair material. Meanwhile, the resulting film maintained the original lamellar structure and suitable water absorption. Cytocompatibility studies showed that the $\beta$-CDDA/OFLX-Col film could promote cell proliferation compared to the control group. Moreover, it is worthy to note that the addition of drugs not only caused no loss of performance, but also showed eligible drug release rate and excellent antibacterial effect. Since the $\beta$-CD-DA/OFLX-Col film possesses suitable physicochemical properties and excellent antibacterial ability, it might provide a new opportunity for bacterial keratitis treatment.

\section{Conflicts of interest}

There are no conflicts to declare.

\section{Acknowledgements}

This study was financially supported by National Natural Science Foundation of China (51603073, 51673071), Guangdong Scientific and Technological Project (2014B090907004),
Natural Science Foundation of Guangdong Province (2017A030313294), Pearl River S\&T Nova Program of Guangzhou (201710010195), the Fundamental Research Funds for the Central Universities.

\section{Notes and references}

$1 \mathrm{WHO}$, Prevention of Blindness and Visual Impairment: Priority Eye Diseases, WHO, New York, 2015.

2 T. L. Du, V. Pillay, Y. E. Choonara, T. Govender and T. Carmichael, Expert Opin. Drug Delivery, 2011, 8, 71-94.

3 D. Achouri, K. Alhanout, P. Piccerelle and V. Andrieu, Drug Dev. Ind. Pharm., 2012, 39, 1599-1617.

4 L. Rabinovich-Guilatt, P. Couvreur, G. Lambert and C. Dubernet, J. Drug Targeting, 2004, 12, 623-633.

5 A. K. Riau, D. Mondal, T. T. Aung, E. Murugan, L. Chen, N. C. Lwin, L. Zhou, R. W. Beuerman, B. Liedberg, S. S. Venkatraman and J. S. Mehta, ACS Biomater. Sci. Eng., 2015, 1, 1324-1334.

6 Y. Liu, L. Ren, K. Long, L. Wang and Y. Wang, Acta Biomater., 2014, 10, 289-299.

7 M. J. Wissink, R. Beernink, J. S. Pieper, A. A. Poot, G. H. Engbers, T. Beugeling, W. G. van Aken and J. Feijen, Biomaterials, 2001, 22, 151-163.

8 J. Chen, X. Shi, L. Ren and Y. Wang, Carbon, 2016, 111, 1827.

9 K. Srinivasan and T. Stalin, Spectrochim. Acta, Part A, 2014, 130, 105-115.

10 R. Krishnan, A. M. Rakhi and K. R. Gopidas, J. Phys. Chem. C, 2012, 116, 25004-25014.

11 S. M. Ali, K. Fatma and S. Dhokale, Beilstein J. Org. Chem., 2013, 9, 1917-1924.

12 M. J. Cornwell, J. B. Huff and C. Bieniarz, Tetrahedron Lett., 1995, 36, 8371-8374.

13 S. Liu, J. Cai, L. Ren, L. Wang and Y. Wang, RSC Adv., 2014, 4, 18608-18611.

14 C. Mu, F. Liu, Q. Cheng, H. Li, B. Wu, G. Zhang and W. Lin, Macromol. Mater. Eng., 2010, 295, 100-107.

15 Z. Xuan, L. Yang, W. Li, L. Kai, W. Lin, S. Liu, Y. Wang and R. Li, Mater. Sci. Eng., C, 2015, 55, 201-208.

16 C. Deng, F. Li, J. M. Hackett, S. H. Chaudhry, F. N. Toll, B. Toye, W. Hodge and M. Griffith, Acta Biomater., 2010, 6, 187-194.

17 C. Zhang, Y. Liang, S. Deng, Z. Wang, R. Li and X. Sun, Clin. Ophthalmol., 2008, 2, 575-579.

18 W. R. Rd, V. Mahaguna and M. Sriwongjanya, Eur. J. Pharm. Biopharm., 1998, 46, 355-360.

19 P. V. Demarco and A. L. Thakkar, J. Chem. Soc., Chem. Commun., 1970, 11, 2-4.

20 A. Guerrero-Martínez, G. González-Gaitano, E. M. Murciano and G. Tardajos, J. Inclusion Phenom. Macrocyclic Chem., 2007, 57, 251-256.

$21 \mathrm{~J}$. Li and X. Zhang, J. Inclusion Phenom. Macrocyclic Chem., 2011, 69, 173-179.

22 W. Liu, C. Deng, C. R. Mclaughlin, P. Fagerholm, N. S. Lagali, B. Heyne, J. C. Scaiano, M. A. Watsky, Y. Kato and R. Munger, Biomaterials, 2009, 30, 1551-1559. 
23 H. Tan, B. Wu, C. Li, C. Mu, H. Li and W. Lin, Carbohydr. Polym., 2015, 129, 17-24.

24 J. Liu, G. Song, Z. Wang, B. Huang, Q. Gao, B. Liu, Y. Xu, X. Liang, P. Ma, N. Gao and J. Ge, Exp. Eye Res., 2007, 84, 599-609.
25 J. B. Fourtillan, J. Granier, B. Saint-Salvi, J. Salmon, A. Surjus, D. Tremblay, M. V. D. Laurier and S. Beck, Infection, 1986, 14, 67-69. 